\title{
Technical Brief: Fatigue Dimples
}

\author{
Arthur Griebel
}

Submitted: 23 January 2009/Published online: 21 March 2009

(C) ASM International 2009

\begin{abstract}
This technical brief is intended to warn failure analysts against assuming that dimples indicate overload fracture. Dimples simply mean that microvoid processes were active; these processes can occur in cyclic as well as monotonic fractures. The failure analyst must examine the entire fracture, the macroscopic as well as the microscopic features, to be confident of the cracking mode.
\end{abstract}

Keywords Fatigue crack growth - Fatigue cracking . Fatigue failure $\cdot$ Fatigue analysis

\section{Fatigue Dimples}

Failure analysis often relies (heavily) on fracture analysis, which entails observing a crack surface and making conclusions about the mode and cause of cracking. Interpreting a crack surface can be complicated by rub, postfracture damage, and corrosion, but in the absence of these obscuring effects the analyst hopes to establish the mode of cracking based on the crack appearance. Once the mode is known, the analyst tries to identify the conditions (material or environmental) that caused the crack.

To successfully follow this chain of deduction from crack appearance backward to cause, the analyst must recognize a correlation between crack appearance and the cracking mode. The correlation is preferably one-to-one, so that an observed crack surface represents only one mode.

At the very least, the analyst wants to distinguish between cracking caused by cyclic loading (fatigue) in which a crack of length $a$ extends incrementally ( $d a / d N)$

A. Griebel ( $\square)$

Stork Climax Research Services, Inc., Wixom, MI, USA

e-mail: beena.nayar@stork.com with each stress cycle $N$, and cracking caused by overload, in which the crack traverses a large distance in a single stress event. Distinguishing between fatigue and overload in ductile materials is frequently based on the presence of striations or dimples, which often are associated with fatigue and overload, respectively. Actually, fatigue does not always produce striations, and dimples are not exclusively associated with overload. The first fact is well recognized, but the second is not, and fatigue dimples are seldom mentioned in the literature. (Becker and Lampman discuss fatigue dimples as "cyclic microvoid processes" on p 578 of Failure Analysis and Prevention, Vol 11 of the ASM Handbook, published by ASM International in 2002. Fatigue dimples are also mentioned on p 53 of Fractography, Vol 12 of the ASM Handbook, published by ASM International in 1987. Other references can be found, but many people engaged in fracture analysis tend to overlook or discount the possibility of dimples appearing on fatigue fracture surfaces.)

An experienced analyst recognizes that fatigue crack morphology changes considerably with propagation rate, $d a / d N$. Striations form only in a limited range of crack propagation rates, and only in some materials. At lower propagation rates the appearance can range from crystallographic to "ropey," and at higher propagation rates fatigue dimples can form.

The following sequence of fractographs demonstrates the range of fatigue crack appearances observed on the fracture surface of a single small screw. Fatigue cracking began in the thread root, and the crack propagated through about $1.6 \mathrm{~mm}$ of the $3 \mathrm{~mm}$ cross section before the remaining material fractured because of overload. Figure 1 is an overview of the crack initiation site. Figures 2 through 11 were obtained at increasing distances from the crack initiation site. 


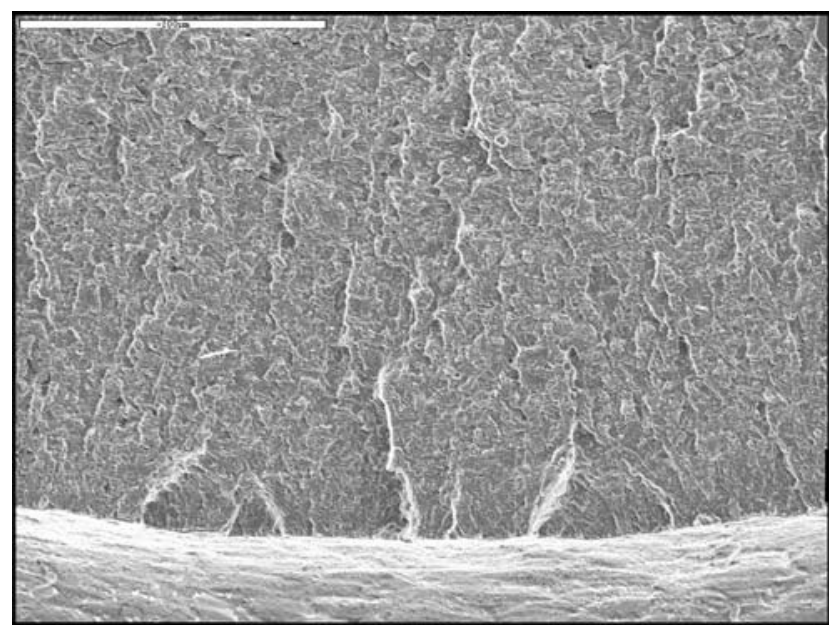

Fig. 1 Fatigue cracking initiated in the thread root at the bottom of this image. The crack propagated from the bottom toward the top of the image. All subsequent images maintain the same orientation as this image

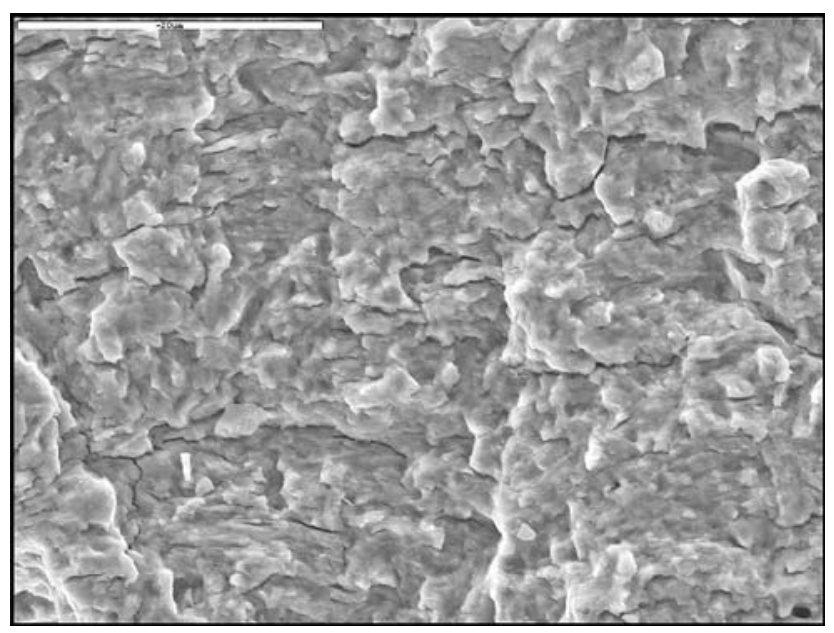

Fig. 2 Crack appearance $0.1 \mathrm{~mm}$ from the crack initiation site

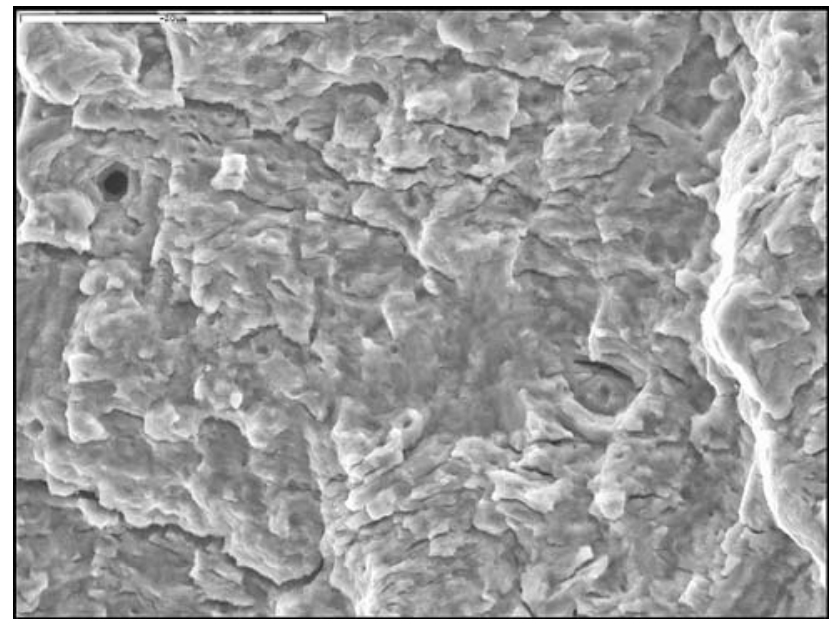

Fig. 3 Crack appearance $0.2 \mathrm{~mm}$ from the crack initiation site

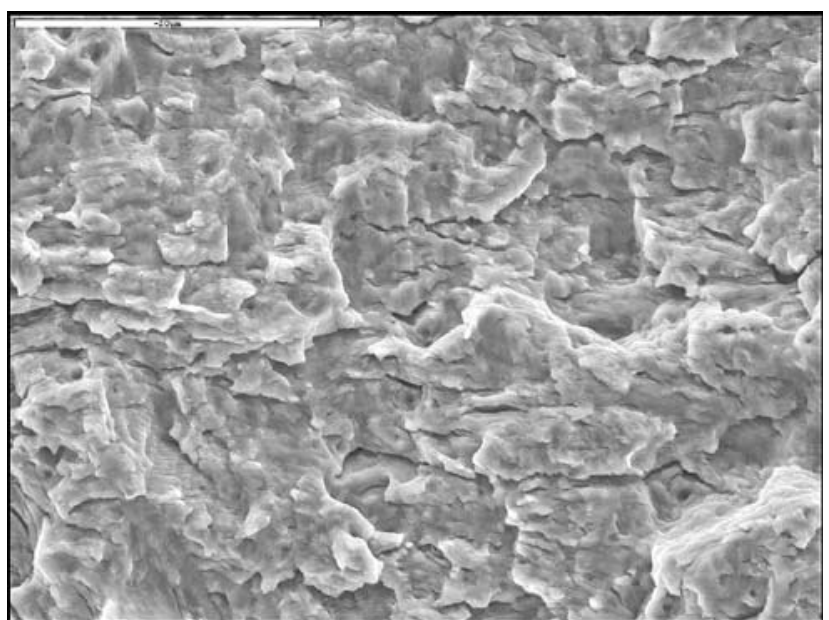

Fig. 4 Crack appearance $0.4 \mathrm{~mm}$ from the crack initiation site

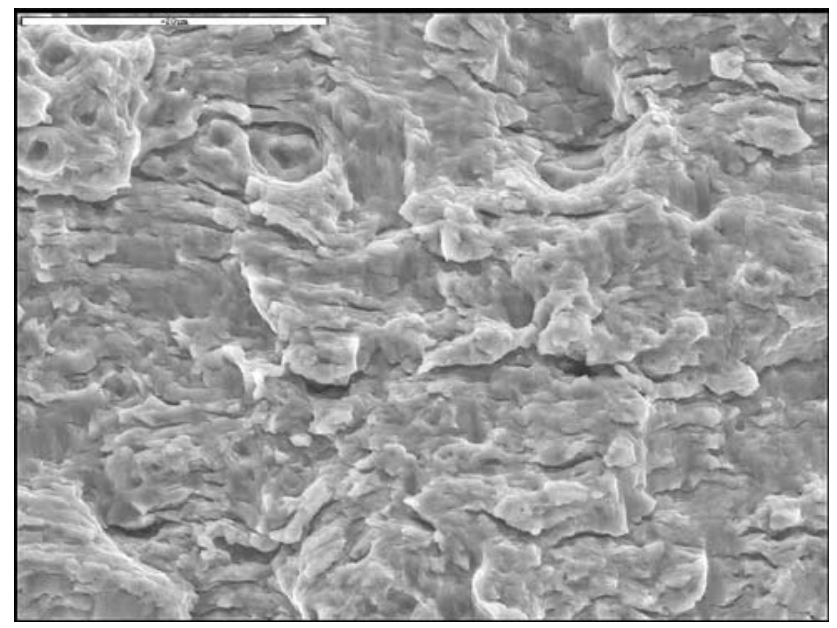

Fig. 5 Crack appearance $0.6 \mathrm{~mm}$ from the crack initiation site

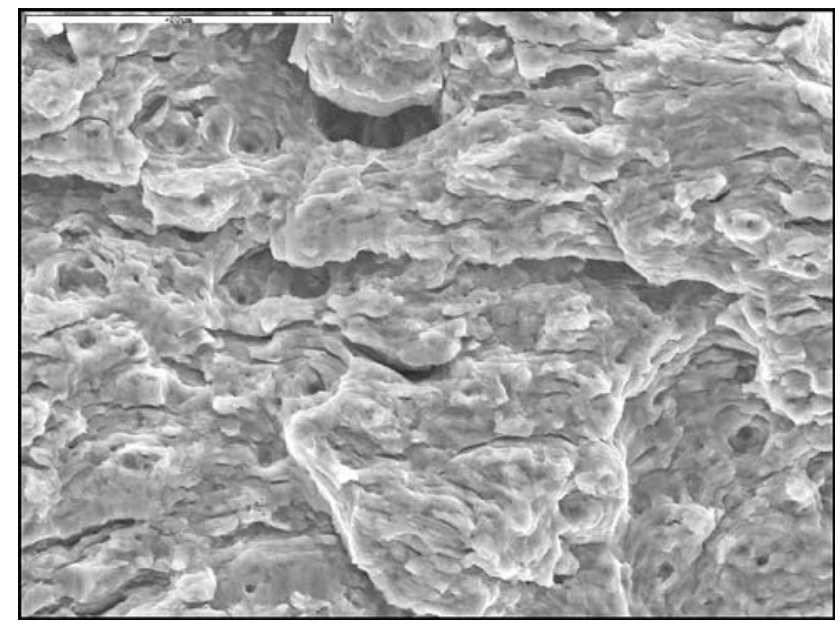

Fig. 6 Crack appearance $0.8 \mathrm{~mm}$ from the crack initiation site 


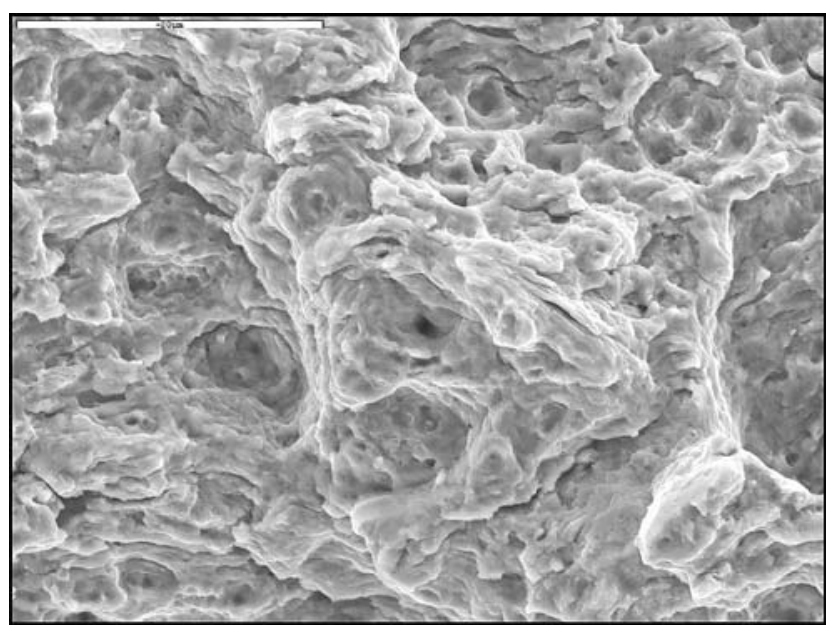

Fig. 7 Crack appearance $1.0 \mathrm{~mm}$ from the crack initiation site

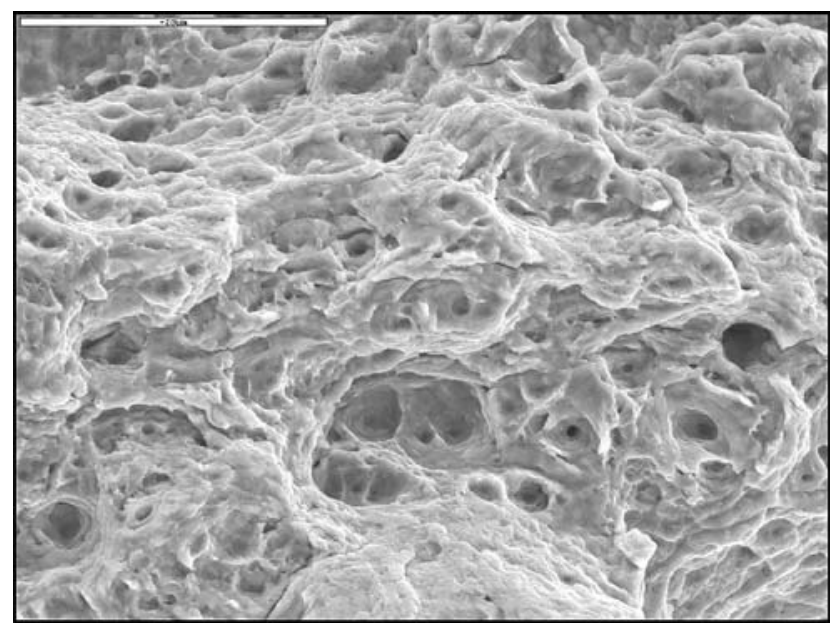

Fig. 8 Crack appearance $1.2 \mathrm{~mm}$ from the crack initiation site

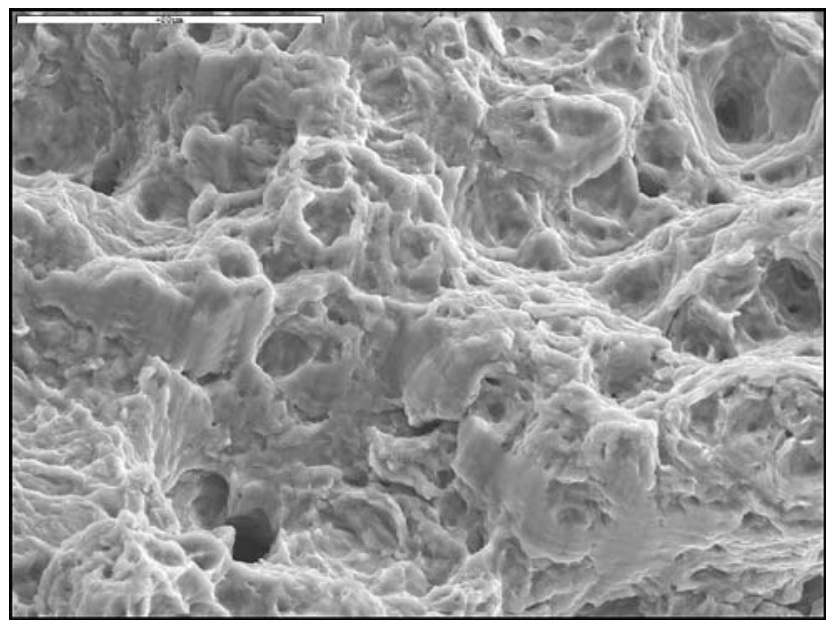

Fig. 9 Crack appearance $1.4 \mathrm{~mm}$ from the crack initiation site

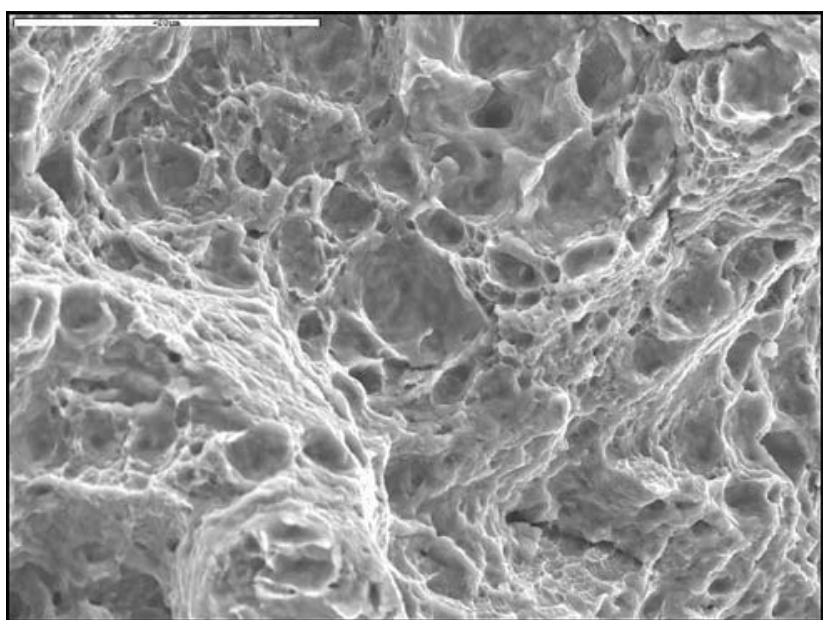

Fig. 10 Crack appearance $1.6 \mathrm{~mm}$ from the crack initiation site

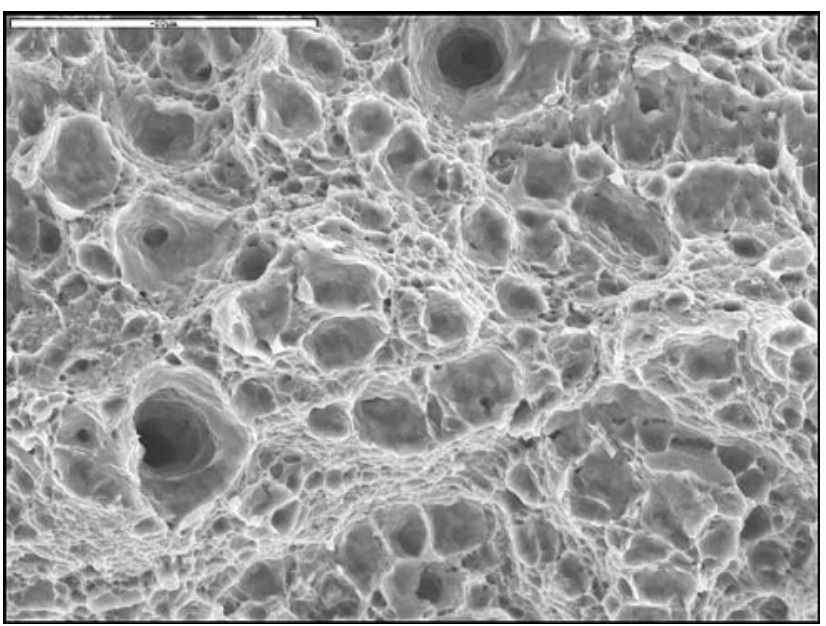

Fig. 11 Crack appearance $1.8 \mathrm{~mm}$ from the crack initiation site

The crack propagation rate, daldN, increased as the crack lengthened and, consequently, the crack surface appearance changes continuously between the crack initiation site and the region of overload. Striation cracks observed in Fig. 2 to 7 leave no doubt that fatigue is the cracking mode in this area. However, there is an increasing amount of "dimplelike" character on the surfaces shown in Fig. 5 to 7 . The regions shown in Fig. 8 and 9 consist mostly of poorly formed dimples with blunt edges; these might be interpreted as dimple rupture caused by overload if they were not part of the sequence of images that clearly shows the crack began as fatigue.

Figure 10 shows dimples with slight blunting of the edges that might arguably be fatigue or overload, with proper classification dependent on macroscopic appearance of the fracture or other evidence. Figure 11 shows wellformed dimples with sharp edges that were observed in the region of final fracture. This is what classic tensile dimples look like. 
This screw exhibited a rather wide range of fatigue appearances; not all fatigue fractures display such a wide variation in morphology. Sometimes a crack comprises mostly one type of surface, such as the poorly formed dimples shown in Fig. 8. It is important for a fracture analyst to examine the entire fracture surface and consider the macroscopic as well as the microscopic evidence of fracture mode. Otherwise, the microscopic fracture morphology can lead to incorrect conclusions about the failure mode. 